\title{
Comparison of Group CBT with Memory Specificity Training (MEST) and Self-distancing \& Group CBT with MEST among Depressed Outpatients
}

\author{
Article by Peter John Sabatelli \\ Ph.D Clinical Psychology, Texila American University \\ Email: psabatelli@btinternet.com
}

\begin{abstract}
Memory specificity training (MEST) or self-distancing (SD) has been associated with improving symptoms of depression. The aim was to compare the efficacy of group CBT with MEST and SD (group A) with group CBT with MEST (group B) in depressed adults and establish the relationships between memory specificity improvement and changes in low mood (BDI-II), rumination (RRS), problem solving (PSI) and suppression (WBSI). Method was to recruit participants from advertisements, 120 fulfilled the inclusion criteria, consented and block randomised to either group. Fifty-five participants from each group $(N=110)$ completed treatment. Results from parametric tests showed group A was statistically significant on all dependent variables, memory specificity (AMS), BDI-II, RRS, PSI, WBSI compared to group $B$ at post-treatment and three month follow-up. Group A was clinically significant on the BDI-II score sat post-treatment. Test of association at three months follow-up showed AMS improvements was independent of improvements in all the other dependent variables $A$ multiple regression analysis confirmed that only RRS had a significant mediating effect on AMS changes. Discussion, group A was more effective for depression than group B, indicating that SD enhanced both MEST and CBT. That AMS may improve mood partly by directly improving rumination. Limitations, not fully blind, no independent therapists, low frequency of supervision checking manual adherence, no SCID, follow-up period short and reduced generalis ability. Future research could repeat this study by recruiting participants from mental health clinics, improve blinding, use SCID, frequent supervision and independent therapists.
\end{abstract}

Keywords: Cognitive Behavioural Therapy, Memory Specificity Training, Self-Distancing.

Contemporary standard cognitive behavioural therapy (CBT) appears to be less effective for depression than classical CBT conducted decades ago [1-2]. It may also be no more effective than other types of psychological therapies for depression [3] including third wave CBT therapies [4]. It is imperative to continue to explore other psychological options that could enhance CBT, such as using memory specificity training (MEST) and self-distancing (SD) as adjuncts to group CBT.

\section{Literature review}

Promising results have been achieved from studies using four to five sessions of MEST that targets over general memory (OGM) by increasing memory specificity (AMS) may or can improve mood [5-7], including rumination, experiential avoidance and problem-solving skills [5]. Over general autobiographical memories (OGM) are strongly associated with making the depression more severe, delays recovery and is a vulnerability factor for subsequent depression [8]. They are defined as a summary of several similar events or a memory of a general time period, spanning more than one day [9]. Memory specificity is a memory for a specific occasion, event that occurred at least one week or longer at a particular place and time within a 24 hour period [5-6].

Theoretically, OGM may be a failure to progress down a memory hierarchy to lower more specific levels of memory during memory retrieval and remain at the general level The capture of memory brings together OGM's that causes or keeps the depression in place. This 
may occur in rumination [10]. Studies that have induced a reduction in rumination led to an increase in AMS, indicating an association between rumination and OGM [11-12] which may be bi-directional [13] but a recent study found no such relationship between rumination and OGM [14].

Access to previously successful problem solving strategies stored in memory to deal with current problems could be captured at the intermediate level of the hierarchy, or at the OGM level [9], poor problem solving is associated with depression and OGM [15].

Increases in OGM are made worse by functional avoidance of specific details of distressing memory events [10] such as suppression. Recalling OGM's is considered as a way to avoid unwanted vivid mental images and strong negative affect that accompanies AMS [16] as seen in depression [17]. Suppression is defined as the effort not to think a specific thought [18], feeling, emotion, urge, sensations or memories, increasing them instead. It increases the risk of depression and makes it more severe [19].

Interesting results have also been reported in self-distancing (SD) induction studies that involved both distancing and reconstruing. Those who were induced to SD felt less distress than those who self-immersed. They focused less on recounting, rumination and more on reconstruing, problem solving that promoted new insights, meanings, improved mood and sense of closure [20-25].

A self-distanced perspective is grounded in social and cognitive psychology theories, including construal level theory and psychological distance. The more distant an object is from the individual, the more abstract, conceptual, non-specific it will be thought of, while the closer the object is, the more precise, concrete it will be thought of [26].

Self-distancing involves taking a mental step back, recalling or retrieving past experiences from the perspective of a distanced observer, a stance that permits a broader context to be observed, allowing and facilitating reconstruing to take place, that may lead to the development of adaptive explanation, understanding, meaning and insight that can reduce current and future distress [20-25; 27]. It is a balance between less recounting and more reconstruing that may account for the emotional regulatory effects of SD [21] especially when using non-first-person pronouns and one's own name than first person pronouns that were associated with self-immersion [25].

Recent studies have proposed expanding the use of MEST, for example in the study by Neshat-Doost et al, 2013, p 7 [6], suggested to use MEST as an ' ....adjunct to CBT..." and Dalgleish et al, 2014, p 8 [28], stated that ' '...MEST may be used as an adjunct...to more comprehensive therapies such as CBT'”

Building on the MEST studies, a larger sample would increase the power of this research, trauma would remain as an exclusion criteria to reduce a possible confounding variable, a balanced group to be randomised, measures to be used to cover not only low mood but also rumination, experiential avoidance and problem solving that was measured in the study by Raes et al [5] but not used in the other two MEST studies [6-7]. There was a need to not only look for difference between groups but also the relationship between AMS changes and other dependent variables, this was attempted in Raes et al study [5] that needs to be repeated and expanded on due to the small sample size and high drop-out rates and non-significant result for experiential avoidance.

This research had a follow up period of three months with strict criteria that participants could only stay in the treatment group if they were not receiving treatment elsewhere. This was required so as to avoid confounding results at three month follow up as occurred in Eigenhuis study [7]. Another novel aspect was the participants were from the United Kingdom (UK) not as I am aware done before.

Self-distancing research mainly covered induction studies using relatively young nonclinically depressed participants [20-25]. Only one study that I was aware of induced SD on a depressed group from San Francisco area in America. There was no extended follow up period, no control group or active comparative group treatment [24], limiting generalisability. In Kross and Ayduk, 2011, p 189 [23], they suggested further research using SD in depressed 
adults. Kross et al, 2012, p566 [24], gave more direction by suggesting whether the benefits of SD studies as above can generalise to depressed adults under a "variety of conditions", that could be extended to cover using SD as an adjunct in an active treatment, such as with group CBT and MEST.

Self-Distancing could enhance a main component of CBT called cognitive reframing, increasing the ability to challenge unhelpful distressing thoughts and then reframe them [29]. It was hypothesised that cognitive distancing (CD) is required to allow engagement with the reframing process [29]. A recent study [30] implied that a key factor to facilitate appraisal and interpretation of one's experience was the use of distancing as found in decentering that can reduce depression [31], similar to the distancing component in SD.

Memory specificity training may be enhanced by facilitating the ability to tolerate, through distancing, strong negative affect [20-25]) associated with the recall of AMS [5-7]. The use of SD may have the effect of increasing AMS more than could be achieved by MEST alone. It may also be able to enhance improvements in mood, rumination and problem solving as seen in Raes et al study [5] and SD studies [20-25].

The purpose of this study was to compare the efficacy of seven sessions with a three month follow up of group CBT with MEST and SD compared to CBT with MEST, building on the need for research using MEST as an adjunct to CBT (Dalgleishet al., 2014, p.8 [28]; NeshatDoost et al., 2013, p.7 [6], and built on the need to incorporate SD with a substantive therapy, group CBT with MEST to see if it could enhance them (Kross and Ayduk, 2011, p 189 [23]; Kross et al, 2012, p 566 [24]. Also it was important to establish any mediating factor(s) between AMS and other dependent variables.

[a] Hypothesis one: There would be a difference in effect between group A and group B on the amount of change of AMS recalled by moderately depressed adults by post- treatment and three month follow up conditions.

[b] Hypothesis two: There would be a difference in effect between group A and group B on mood change in moderately depressed adults by post-treatment and three month follow up

[c] Hypothesis three: There would be a difference in effect between group A and group B on amount of change of rumination in moderately depressed adults by post- treatment and three month follow up

[d] Hypothesis four: There would be a difference in effect between group A and group B on amount of change of problem solving in moderately depressed adults by post-treatment and three month follow up conditions.

[e] Hypothesis five: There would be a difference in effect between group A and group B on amount of change of suppression in moderately depressed adults by post- treatment and three month follow-up. [f] Hypothesis six: There is a relationship of association and mediation between changes in memory specificity and changes in one or more other variables, BDI-II, RRS, PSI and WBSI at three months follow-up.

\section{Method}

A mixed group design, $2 \times 3$ was selected to compare differences in the dependent variables, AMT, RSS, PSI, WBSI between and within group A and group B at each time point from pre-treatment, post-treatment and three month follow-up. A correlational design, followed by a repeated ANOVA with covariates and multiple regression was used, alpha set at 0.05 to test the relationship between improved memory specificity and improved mood, rumination, problem solving and suppression at three month follow up. A randomised controlled trial (RCT) was used as it is a powerful method when comparing the effectiveness of interventions [32] and helps to minimise allocation and confounding variable bias [33].

Data from Raes study [5] suggested a sample size of 22 (11 per group) would provide $80 \%$ power, with a directional alpha of .05 to detect a similar improvement in AMS. The sample size had to be larger than 11 per group as a requirement of 36 or more for each treatment group was needed to achieve medium effect sizes averaging $\mathrm{d}=0.51$ in previous research on self-distancing [20-21]. 
Two treatment manuals was developed, one for group A and one for group B, ethical approval was obtained then the research was advertised in two London boroughs. Prospective participants responded by e-mail to leaflets posted through the letterbox of people's homes, flyers posted on the advertisement boards in supermarkets, health clubs, local libraries, community centres, religious places of worship, several newsagents and charity shops. For each cohort there was a screening process, participants who scored moderately or high on each questionnaire got interviewed to ensure they fulfilled the DSM 5 criteria for depression [34], met the inclusion and exclusion criteria and gave written consent.

They were then cluster randomised using a stratified randomisation procedure [35]. Sixty participants for each group were initially recruited, 55 for each group started and completed the treatment. The confidentiality of participants was protected by storing all data on an assigned participant number rather than their name or other identifying data. The participants were made aware that involvement in the study was voluntary and withdrawal from the study was acceptable without repercussions.

Inclusion criteria included, aged between 20-60, Beck depression inventory (BDI-II) score of 20 or more but less than 29 [36] and fulfil DSM-5 criteria for depression. Has an AMS of less than 0.70 as assessed on the autobiographical memory test[37]. Exclusion criteria, high levels of suicidality or harm to self, primary bipolar, or psychotic, anxiety, personality disorder; current drug, alcohol abuse, dependence, presence of head trauma or organic brain damage, history of childhood abuse; trauma symptoms, chronic pain, adjustment problem, primary problem is anxiety, receiving treatment for depression elsewhere, poor grasp of the English language, reading, writing, unwilling or unable to give written consent.

The autobiographical memory test (AMT) was developed by Williams \& Broadbent, (1986) measures both OGM and AMS and has good validity and reliability [37]. In this research, two parallel sets of 18 cue-words, nine positive and nine negative, similar to a previous MEST study [6], were matched for familiarity and emotionality using three independent raters [38] for pre-test, post and follow up.

Beck's depression inventory (BDI-II) is a 21-item self-report questionnaire used for measuring the severity of depression that has high reliability and validity [36, 39]. Ruminative responses scale (RRS) measures how participants tend to focus on the self, symptoms and reasons, causes for low mood, shows good reliability and validity [40]. Problem-solving inventory (PSI) (Heppner, 1988) is a measure of self-appraised problem solving ability, style [41] that has high reliability [42]. The white bear suppression inventory (WBSI) measures thought suppression, has good internal consistency and stability with a one week test-retest correlation of .92, and a three week to three month test-retest correlation of .69 [43].

The CBT components consisted of behavioural activation and cognitive reframing that followed the Beckian cognitive model and Lewholm behavioural model [44,29]. MEST guidelines was followed $[5,6]$ but spread over seven sessions instead of five due to having to accommodate the CBT content and self-distancing technique [20-25]. For group B it had exactly the same content, format as for group A except no SD was done, each session lasted 90 minutes that was carried out in a community private health centre on the same day. Both manuals where scrutinised by other professionals to ensure they were the same except for SD in group A and that the CBT components were typical of what was used for depression in the UK.

Changes in the dependent variables are the measurement scores on the AMT, BDI-II, RRS, PSI, WBSI, interval data at the various time conditions, pre, post treatment and follow up. The continuous variables all met the normality test and met the conditions necessary for the specific parametric tests described below to test hypotheses one to six.

Significance level for the hypothesis tests was set at .01 with a confidence level of $99 \%$ for all statistical tests. Effect sizes are indicated in terms of both Cohen's $d$ and partial $\eta^{2}$, the former has effect sizes of 0.2, 0.5, and 0.8 that are considered small, medium, and large [47]. To treat with caution, for partial $\eta^{2}$ it was suggested that the effect size in a one way ANOVA may be $0.01,0.06$ and 0.06 , respectively small, medium and large. For repeated measurement 
such as repeated ANOVA's, partial $\eta^{2}$ could be $0.01,0.09$ and 0.25 , corresponding to small, medium and large effects [45]. All effect sizes are reported as significant at $\mathrm{p}<.01$.

Differences in categorical variables such as gender, marital status, education, occupation status, ethnicity and religion was tested with the chi square test. Continuous variables such as age, was tested using a univariate ANOVA. Using SPSS 22 software, repeated MANOVA, univariate ANOVA with Bonferroni correction, independent t-tests answered hypotheses one to five. Hypothesis six was tested using the Pearson product-moment correlation coefficient, partial correlations, repeated ANOVA and multiple regression analysis. To help guarantee the integrity of each treatment, two random audio recordings and two random in situ observations were made between session two to seven by an independent clinical psychologist. This was to check for manual adherence and bias in non-verbal presentation, none was found.

\section{Results}

Missing data was low for both groups (i.e., 8.3\% at post-treatment and one-month followup), the decision was made to not utilize multiple imputation strategies to replace missing values. SPSS 22listwise output was relied on as the percentage of $8.3 \%$ does not compromise statistical analysis [46]. At pre-treatment a chi-square test of demographic categorical variables for both groups and univariate ANOVA for age range showed no statistical difference. All dependent variable scores between the two groups at pre-treatment also had no statistical significant differences.

For group A and group B pre-treatment data was normal with no outliers.. Multicollinearity was met; the box's test of equality of covariance matrices $(p=.024)$ indicated that the assumption of homogeneity of variance-covariances matrices was also met. Levene test for the equality of variances for each group at each time points were largely met. A repeated within and between groups multivariate analysis of variance (RMANOVA) was performed to investigate the differences between group $A(N=55)$ and group $B(N=55)$. The combined dependent variables used, AMS, BDI-II, RRS, PSI and WBSI. The independent variables was treatment and time, $2 \times 3$ design, pre-treatment, post-treatment and three month follow up. Preliminary assumption testing was conducted to check for normality, univariate and multivariate outliers, homogeneity of variance matrices, and multicollinearity, with no serious violations noted.

There was a statistically significant differences between both groups on the combined dependent variables at post-treatment, $\mathrm{F}(5,104)=161.458, \mathrm{p}=.0001$; Pillai trace $=.886$; partial eta squared $=.886$; and also at three month follow up, $\mathrm{F}(5,104)=427.719, \mathrm{p}=.0001$, Pillai Trace $=.954$, partial eta squared .954 . A statistical significant difference within groups was found at each time point by post treatment, $\mathrm{F}(5,104)=243.474, \mathrm{p}=.0001$, Pillai Trace $=$ .921 , partial eta squared $=.921$; and also at three month follow up, $F(4,104)=148.157$, p= .0001 ; Pillai Trace $=.954$; partial eta squared $=.954$. The results for the dependent variables AMS, BDI-II, RRS, PSI and WBSI was considered separately using a univariate ANOVA with a Bonferroni adjustment alpha level of .001. There was statistical significance found between both groups at post-treatment and three month follow up with large effect sizes- see tables 1 and 2 .

Table 1 Univariate ANOVA: between group results for each dependent variable at post-treatment

\begin{tabular}{lllll}
\hline Hypothesis & DV & $\mathrm{df}=\mathrm{F}$ & $\mathrm{p}$ & partial $\eta^{2}$ \\
\hline One & AMS & $(1,108)=16.251$, & $<.0001$ & .131 \\
Two & BDI-II & $(1,108)=406.635$ & $<.0001$ & .790 \\
Three & RRS, & $(1,108)=167.380$ & $<.0001$ & .608 \\
Four & PSI & $(1,108)=258.547$ & $<.0001$ & .705 \\
Five & WBSI & $(1,108)=280.693$ & $<.0001$ & .722 \\
\hline
\end{tabular}

$\mathrm{DV}=$ dependent variable; $\mathrm{df}=$ degrees of freedom; $\mathrm{F}=$ distribution; $\mathrm{p}=$ significance level 
Texila International Journal of Psychology

Volume 1, Issue 2, Dec 2016

Table 2 Univariate ANOVA: between group results for each dependent variable at three month FU

\begin{tabular}{lllll}
\hline Hypothesis & $\mathrm{DV}$ & $\mathrm{df}=\mathrm{F}$ & $\mathrm{p}$ & partial $\eta^{2}$ \\
\hline One & AMS & $(1,108)=44.619$ & $<.0001$ & .292 \\
Two & BDI-II & $(1,108)=472.582$ & $<.0001$ & .879 \\
Three & RRS, & $(1,108)=516.389$ & $<.0001$ & .789 \\
Four & PSI & $(1,108)=1101.846$ & $<.0001$ & .911 \\
Five & WBSI & $(1,108)=617.685$ & $<.0001$ & .851 \\
\hline
\end{tabular}

$\mathrm{DV}=$ dependent variable; $\mathrm{df}=$ degrees of freedom; $\mathrm{F}=$ distribution; $\mathrm{p}=$ significance level

An independent t-test was conducted to determine were the differences in AMS, BDI-II, RRS, PSI and WBSI scores lay between group A and group B.

Hypothesis one was answered by showing that group A had greater improvement on the AMS scores $(\mathrm{M}=91.22, \mathrm{SD}=2.28)$ than group $\mathrm{B}(\mathrm{M}=88.05, \mathrm{SD}=3.3)$, a statistically significant mean difference of 3.17 (99\% CI, 1.70 to 4.63$), \mathrm{t}(108)=5.675, \mathrm{p}=.0001, \mathrm{~d}=1.09$ at posttreatment. Group A had greater improvement on the AMS scores $(\mathrm{M}=96.58, \mathrm{SD}=2.41)$ than group $B(M=92.54, S D=2.55)$, a statistically significant mean difference of 4.02 (99\% CI, 2.80 to 5.28), $\mathrm{t}(107.65)=8.550, \mathrm{p}=.0001, \mathrm{~d}=1.64$ at three month follow up.

Hypothesis two was answered by showing that group A had greater improvement on the BDI-II scores $(\mathrm{M}=8.18, \mathrm{SD}=1.15)$ than group $\mathrm{B},(\mathrm{M}=14.04, \mathrm{SD}=0.77)$, a statistically significant mean difference of -5.86 (99\% CI: -6.33 to -5.37$)$, t $(95.48)=-31.889, \mathrm{p}=.0001$, $\mathrm{d}=6.10$ at post-treatment. Group A had greater improvement on the BDI-II scores $(\mathrm{M}=3.75$, $\mathrm{SD}=1.72)$ than group $\mathrm{B}(\mathrm{M}=10.60$,

$\mathrm{SD}=1.42)$, a statistically significant mean difference of -6.85 (99\% CI, -7.65 to -6.06$), \mathrm{t}$ $(108)=-22.249, \mathrm{p}=.0001, \mathrm{~d}=4.34$ at three month follow up.

Hypothesis three was answered by showing that group A had greater improvement on the RRS $(M=25.20, S D=1.21)$ than group $B(M=32.51, S D=1.44)$, a statistically significant mean difference of -7.31 (99\% CI, -7.97 to -6.65$)$, $\mathrm{t}(108)=-28.856, \mathrm{p}=.0001, \mathrm{~d}=5.50$ at posttreatment. Group A had greater improvement on the RRS $(\mathrm{M}=23.34, \mathrm{SD}=1.07)$ than group $\mathrm{B}$ $(\mathrm{M}=29.07, \mathrm{SD}=0.99)$, a statistically significant difference of -5.73 (99\% CI:-6.35 to -5.32$), \mathrm{t}$ $(108)=-29.587, \mathrm{p}=.0001, \mathrm{~d}=5.61$ at three month follow up.

Hypothesis four was answered by showing that group A had greater improvement on the PSI $(M=74.89, S D=1.81)$ than group $\mathrm{B}(\mathrm{M}=86.78, \mathrm{SD}=1.99)$, a statistically significant mean difference of $-11.89(99 \% \mathrm{CI},-12.84$ to -10.94$)$, $\mathrm{t}(108)=-32.700, \mathrm{p}=.0001, \mathrm{~d}=6.25$ at posttreatment. Group B had greater improvement on the PSI $(\mathrm{M}=55.58, \mathrm{SD}=2.32)$ than group B $(\mathrm{M}=75.56, \mathrm{SD}=2.96)$, a statistically significant mean difference of -19.38 (99\% CI, -21.31 to -18.65), $\mathrm{t}(108)=-39.425, \mathrm{p}=.0001, \mathrm{~d}=7.51$ at three month follow up.

Hypothesis five was answered by showing that group A had greater improvement on the WBSI $(M=23.27, S D=1.25)$ than group $B(M=32.33, S D=1.23)$, a statistically significant difference of $-9.06(99 \% \mathrm{CI}, 9.68$ to -8.43$)$, $\mathrm{t}(108)=-38.182, \mathrm{p}=.0001, \mathrm{~d}=7.31$ at posttreatment. Group A had greater improvement on the WBSI $(\mathrm{M}=19.38, \mathrm{SD}=1.10)$ than group $\mathrm{B}(\mathrm{M}=27.00, \mathrm{SD}=1.12)$, a statistically significant difference of -7.62 (99\% CI:-8.17 to $-7.06 \mathrm{t}$ $(108)=-36.003, \mathrm{p}=.0001, \mathrm{~d}=7.62$ at three month follow up.

Hypothesis six was answered through tests of association, repeated ANOVA and mediational analysis. There was moderate to large correlations that were statistically significant found between all variables at three month follow up. A partial correlation was run to determine the relationship between AMS and RRS at three months follow-up, while controlling for BDI-II scores. There was a small to moderate negative partial correlation between AMS (94.56 \pm 3.20$)$ and RRS (26.15 \pm 3.10$)$, whilst controlling for BDI-II (7.17 \pm $3.78)$, that was statistically significant, $(\mathrm{r}(107)=-.289, \mathrm{~N}=110, \mathrm{p}<.002$. However, zeroorder correlations showed a statistically significant, large, negative correlation between AMS and RRS $(\mathrm{r}(108)=-.612, \mathrm{n}=110, \mathrm{p}<.001)$, indicating that the BDI-II changes had a significant influence in controlling the relationship between AMS and RRS. 
A partial correlation was run to determine the relationship between AMS and PSI at three months follow-up whilst controlling for BDI-II scores. There was a small to moderate negative partial correlation between AMS $(94.56 \pm 3.20)$ and PSI $(65.57 \pm 10.37)$, whilst controlling for BDI-II $(7.17 \pm 3.78)$, that was statistically significant, $(\mathrm{r}(107)=-.255, \mathrm{~N}=$ $110, \mathrm{p}<.007)$. However, zero-order correlations showed a statistically significant, large, negative correlation between AMS and PSI $(\mathrm{r}(108)=-.603, \mathrm{~N}=110, \mathrm{p}<.001)$, indicating that the BDI-II changes had a significant influence in controlling the relationship between AMS and PSI.

A partial correlation was run to determine the relationship between AMS and WBSI at three months follow-up whilst controlling for BDI-II scores. There was a small to moderate, negative partial correlation between AMS $(94.56 \pm 3.20)$ and WBSI $(23.19 \pm 3.98)$, whilst controlling for BDI-II $(7.17 \pm 3.78)$, that was statistically significant, $(\mathrm{r}(107)=-.276, \mathrm{~N}=$ $110, \mathrm{p}<.004)$. However, zero-order correlations showed a statistically significant, large, negative correlation between AMS and WBSI $(\mathrm{r}(108)=-.613, \mathrm{~N}=110, \mathrm{p}<.001)$, indicating that the BDI-II changes had a significant influence in controlling the relationship between AMS and WBSI.

A repeated ANOVA was run on the changes in AMS from pre-treatment to three months follow-up, the within-subject factor. The covariates used were BDI-II, RRS, PSI, and WBSI to find out if improved changes in these variables had an effect on improved changes on the AMS variable or not. A repeated ANOVA was run to establish the baseline statistic for the AMS at three months follow up before doing the covariates. There was a statistically significant AMS score change, with improvement from pre-treatment to three months followup, $\mathrm{F}(1,109)=8439.424, \mathrm{p}<.001$, partial $\eta 2=.987$. Running the same test using the BDI-II as the covariate, the AMS remained significant and the effect size large but substantially reduced, $\mathrm{F}(1,107)=21.012, \mathrm{p}<.0001$, partial $\eta 2=.164$. The RRS was then run as the covariate, and the AMS remained statistically significant with a reduced but still large effect size, $\mathrm{F}(1,107)=33.456, \mathrm{p}<.0001$, partial $\eta 2=.238$. The PSI was run as the covariate, and the AMS remained statistically significant with a reduced but still large effect size, $F(1,107)=$ $17.218, \mathrm{p}<.0001$, partial $\eta 2=.139$. The WBSI was run as the covariate, and the AMS remained statistically significant with a reduced but still large effect size, $F(1,107)=53.777$, $\mathrm{p}<.0001$, partial $\eta 2=.334$.

The repeated ANOVA results established that improved changes in AMS remained statistically significant after controlling for improved change scores on the BDI-II, RRS, PSI, and WBSI that indicates the changes in these covariates do not explain how the changes in AMS occur.

A multiple linear regression was calculated to determine the amount of variance of the dependent variable AMS that is explained by each predictor variable individually. The predictors included BDI-II, RRS and PSI. There was reasonable linearity as assessed by partial regression plots and a plot of studentized residuals against the predicted values. There was independence of residuals, as assessed by a Durbin-Watson statistic of 2.325. There was reasonable homoscedasticity, as assessed by visual inspection of a plot of studentized residuals versus unstandardized predicted values. There were nostudentized deleted residuals greater than \pm 3 standard deviations, no leverage values greater than 0.2 , and values for cook's distance above 1 . There assumption of normality was met, as assessed by Q-Q Plot. Tolerance for the RRS was .181, PSI .144 and BDI-II .210. The overall model was significant, F (3, $106)=22.139, \mathrm{p}<.001, \mathrm{R}^{2}=.385$, accounting for $38.5 \%$ of the variance. The results indicated that RSS was the only significant predictor of increased AMS scores and the BDI-II and PSI were not significant predictors of change in AMS scores.

A multiple linear regression was calculated to determine the amount of variance of the dependent variable WBSI that is explained by each predictor variable individually. There was independence of residuals, as assessed by a Durbin-Watson statistic of 2.014 and reasonable homoscedasticity, as assessed by visual inspection of a plot of studentized residuals, versus unstandardized predicted values. There were no studentized deleted residuals greater than \pm 3 
Texila International Journal of Psychology

Volume 1, Issue 2, Dec 2016

standard deviations. There assumption of normality was met, as assessed by Q-Q Plot. Tolerance for the AMS was .610, BDI-II .221, RRS .159 and PSI .136.

Table 4 Multiple Regression Analyses of BDI-II, RRS and PSI on AMS scores (N=110)

\begin{tabular}{lllll}
\hline Variable & $\mathrm{B}$ & $\mathrm{SE}_{\mathrm{b}} \beta$ & $\mathrm{p}$ & \\
\hline Intercept & 107.303 & 5.786 & & \\
BDI-II & -0.173 & 0.140 & -0.205 & \\
RRS & -0.466 & 0.184 & -0.453 & $0.013^{*}$ \\
PSI & -0.009 & 0.102 & 0.017 & 0.934 \\
\hline
\end{tabular}

Note: ${ }^{*} \mathrm{p}<.05 ; \mathrm{B}=$ unstandardized regression coefficient; $\mathrm{SE}_{\mathrm{b}}=$ Standard error of the coefficient; $\beta=$ standardized coefficient;

Table 5 Multiple Regression Analyses of AMS, BDI-II, RRS and PSI on WBSI scores (N=110)

\begin{tabular}{llllll}
\hline Variable & $\mathrm{B}$ & $\mathrm{SE}_{\mathrm{b}} \beta$ & $\mathrm{T}$ & $\mathrm{p}$ & \\
\hline Intercept & 3.541 & 4.978 & & & \\
AMS & -0.036 & 0.044 & -0.029 & -0.822 & 0.413 \\
BDI-II & 0.208 & 0.062 & 0.198 & 3.368 & $0.001^{*}$ \\
RRS & 0.312 & 0.089 & 0.243 & 3.512 & $0.001^{*}$ \\
PSI & 0.205 & 0.029 & 0.533 & 7.106 & $0.001^{*}$ \\
\hline
\end{tabular}

Note: ${ }^{*} \mathrm{p}<.05 ; \mathrm{B}=$ unstandardized regression coefficient; $\mathrm{SE}_{\mathrm{b}}=$ Standard error of the coefficient; $\beta=$ standardized coefficient

The predictors included AMS, BDI-II, RRS and PSI. The overall model as significant, F $(4,105)=301.059, \mathrm{p}<.001, \mathrm{R}^{2}=.920$, accounting for $92 \%$ of the variance. The results indicated that BDI-II, RRS and PSI were significant predictors of decreased WBSI scores. The AMS was not a significant predictor of decreased WBSI scores. The result in table 5suggests that WBSI is not a significant predictor of change in AMS scores but was for the BDI-II, RRS and PSI.

Table 6 shows reliable clinical significant change on BDI-II scores for group A compared to group B.

Table 6 Reliable clinical change on the BDI-II at post-treatment and three months follow-up

\begin{tabular}{llllll}
\hline Group & Pre: BDI-II & Post: BDI-II & 3mth FU & Change & 99\%CI:Clinical Significance \\
\hline A & 26.3 & 8.2 & & 18.1 & 13.59 (achieved) \\
& & & 3.7 & 22.6 & 13.59 (achieved) \\
B & \multirow{2}{*}{26} & \multirow{2}{*}{14} & & 12 & 13.59 (not achieved) \\
& & & 10.6 & 15.4 & 13.59 (achieved) \\
\hline
\end{tabular}

Note: input used- SD=11.8, test-retest=0.90 (Steer et al., 1999); (Devilly, 2004) . http://www.swin.edu.au/victims

\section{Discussion}

Results for hypothesis one to six rejected the null hypothesis and accepted the alternative hypothesis with group A showing greater improvement in all the dependent variables, AMS, BDI-II, RRS, PSI and WBSI at post-treatment and three month follow-up compared to group B. The results here supported Raes et al [5] findings that AMS improvements occurred independently of improvements in BDI-II, PSI and WBSI scores only but not for RRS. The multiple regression analysis suggested that the BDI-II, RRS, PSI and WBSI together could account for up to $40 \%$ of the variance in change in the AMS scores but only the RRS could account for almost half that improvement making the RRS a significant predictor of AMS score changes.

This result clarifies the assertions in previous MEST studies that AMS gains increase improvements in depression, namely improved mood [5-7]). Group A may have enhanced AMS improvement by increasing improvement in RRS, a mediating factor for AMS, confirming previous experimental studies [11-12]. Alternatively, improvement in RRS score 
may have been due to a possible bi-directional relationship with AMS improvement [13], more so than group B.

Self-distancing as an adjunct as in group A did improve the outcomes of CBT more so than MEST which restricted itself to improving AMS that was not associated with mediating improvements in the other dependent variables, except possibly having a small reciprocal mediating effect in improving rumination and in turn improving mood .

The addition of SD to group CBT and MEST enhanced both their outcomes and would be a more clinically useful tool as an adjunct with CBT and MEST to use to treat moderate depression within the National Health Service in the United Kingdom and elsewhere than just group CBT with MEST.

Limitations included, study was not fully blind, no independent therapists used to run groups, no SCID used. All participants were highly motivated as evidenced by voluntary participation, high attendance rate, high homework compliance between both groups, factors not typical of clinically depressed adults that attend out-patient clinics for CBT. There is limited generalisability due to participants being primarily depressed, co clinical co-morbidity and not on anti-depressant medication. The researcher running both group interventions did not receive training to use the treatment manuals for group A and group B, compiled though by the researcher. This researcher also did not receive weekly supervision to check manual adherence when running the groups. This was limited to a few random audio recordings and direct observations by an independent experienced clinician.

\section{Conclusion}

The findings of this study addressed the stagnation in outcomes for moderate depression [1-2] and expanded the work of previous researchers in the area of MEST for depression [5-7] and SD [20-25]. Both groups can improve depression symptomatology such as low mood, rumination, problem solving and suppression but only group A compared to group B on all dependent variables at all time points was more efficacious, not only statistically but clinically significant as well for moderate depression.

The research helped to clarify that improvement in AMS was mediated by improvement in rumination which in turn could improve mood which could explain why in the previous MEST studies [5-7] they found an association between improvement AMS and mood. This research can be repeated with modifications, by having a larger sample size, better blinding, using SCID, regular independent supervision for each deliverer of group intervention; using independent and different therapists to run each group intervention. Participants can be recruited from typical mental health out-patient clinics with co-morbidity.

\section{References}

[1]. Aldao, A., Nolen-Hoeksema, S \& Schweizer, S. (2010). Emotion-regulation strategies across psychopathology: a meta-analytic review. Clinical Psychology Review, 30, 217-237. doi: 10.1016/j.cpr.2009.11.004

[2]. American Psychiatric Association. (2013). Diagnostic and statistical manual of mental disorders (5th ed.). Arlington, VA: American Psychiatric Publishing

[3]. Barth, J., Munder, T., Gerger, H., Nüesch, E., Trelle, S., Znoj, H., ...Cuijpers, P.(2013). Comparative Efficacy of Seven Psychotherapeutic Interventions for Patients with Depression: A Network Meta-Analysis. PLoS Medicine, 10(5), e1001454. doi:10.1371/journal.pmed.1001454.

[4]. Brennan, K., Barnhofer, T., Crane, C., Duggan, D., \& Williams, J. M. G. (2015) Memory Specificity and Mindfulness Jointly Moderate the Effect of Reflective Pondering on Depressive Symptoms in Individuals With a History of Recurrent Depression. Journal of Abnormal Psychology. Advance online publication. http://dx.doi.org/10.1037/abn0000027

[5]. Beck, A. T., Rush, J. A., Shaw, B. F., \& Emery, G. (1979). Cognitive therapy fordepression. New York: Guilford Press. 
Texila International Journal of Psychology

Volume 1, Issue 2, Dec 2016

[6]. Bernstein, A., Hadash, Y., Lichtash, Y, Tanay, G., Shepherd, K., \& Fresco, D.M. (2015). Decentering and Related Constructs A Critical Review and Metacognitive Processes Model. Perspective Psychological Science10(5):599-617

[7]. Beck AT, Steer RA \& Brown GK (1996). Manual for the Beck Depression Inventory-II. San Antonio, TX: Psychological Corporation

[8]. Barlow, D. (2008). Clinical Handbook of Psychological Disorders, Fifth Edition: A Step-by-Step Treatment Manual. The Guildford Press, London

[9]. Cuijpers P, Berking M, Andersson G, Quigley L, Kleiboer A, \& Dobson KS (2013). A metaanalysis of cognitive behavior therapy for adult depression, alone and in comparison to other treatments. Canadian Journal of Psychiatry, 58, 376-385

[10]. Cohen, J. (1992). A Power Primer. Psychological Bulletin, 11, 155-159. doi.org/10.1037/00332909.112.1.155

[11]. Debeer, E., Hermans, D., \& Raes, F. (2009). Associations between components of rumination and autobiographical memory specificity as measured by a Minimal Instructions Autobiographical Memory Test. Memory, 17 (8), 892-903.doi:10.1080/09658210903376243

[12]. D'Zurilla T.J., Chang E.C., Nottingham E.J, \& Faccini L., (1998). Social problem- solving deficits and hopelessness, depression, and suicidal risk in college students and psychiatric inpatients. Journal Clinical Psychology, 1091-1107. doi:10.1002/(SICI)1097-4679(199812)54:8<1091::AIDJCLP9>3.0.CO;2-J

[13]. Dalgleish, T., Bevan, A., McKinnon, A., Breakwell, L., Mueller, V., Chadwick, I. et al (2014). A comparison of MEmory Specificity Training (MEST) to education and support (ES) in the treatment of recurrent depression: study protocol for a cluster randomised controlled trial. Trials. 15. 1-9. doi:10.1186/1745-6215-15-293

[14]. Eigenhuis, E, Seldenrijk, A, van Schaik, A, Raes, F, van Oppen, P. Feasibility and effectiveness of memory specificity training in depressed outpatients: a pilot study. Clinical Psychology and Psychotherapy. 2015, Dec, doi: 10.1002/cpp.1995

[15]. Feneck, O.R., 2009, Clinical research in anaesthesia: randomized controlled trial or observational studies? European Journal of Anaesthesiology 24: 1-5

[16]. Gecht, J., Kessel, R., Forkmann, T., Gauggel, S., Drueke, B., Scherer, A., \& Mainz, V. (2014). A mediation model of mindfulness and decentering: sequential psychological constructs or one and the same? BMC Psychology, 2:18.

[17]. Griffith, J. W., Sumner, J. A., Debeer, E., Raes, F., Hermans, D., Mineka, S., \& . .Craske, M. G. (2009). An item response theory/confirmatory factor analysis of the Autobiographical Memory Test. Memory, 17, 609-623

[18]. Hunot, V., Moore, T.H.M., Caldwell, D.M., Furukawa, T.A., Davies, P., Jones, H., ...Churchill R.(2013). 'Third wave' cognitive and behavioural therapies versus other psychological therapies for depression. Cochrane Database of Systematic 10. Art. No.: CD008704. doi:10.1002/14651858.CD008704.pub2.

[19]. Hermans D, de Decker A, de Peuter S, Raes F, Eelen P, \& Williams JMG: (2008). Autobiographical memory specificity and affect regulation: Coping with a negative life event. Depression Anxiety, 25:787-792. doi:10.1002/da.20326

[20]. Heppner, P. P. (1988). The Problem Solving Inventory. Palo Alto, CA: Consulting Psychologists Press

[21]. Hanna, D. and Dempster, M. (2012). Psychology statistics for dummies. John Wiley and Sons, Ltd, Chichester, West Sussex, England

[22]. Johnsen, T. J., \& Friborg, O. (2015, May 11). The Effects of Cognitive Behavioral Therapy As an Anti-Depressive Treatment is Falling: A Meta-Analysis. Psychological Bulletin. Advance online publication. doi:org/10.1037/bul

[23]. Kross, E., Ayduk, Ö, \& Mischel, W. (2005). When asking 'why' does not hurt: Distinguishing rumination from reflective processing of negative emotions. Psychological Science, 16(9), 709-715. doi:10.1111/j.1467 9280.2005.01600.x 
[24]. Kross, E., \& Ayduk, Ö. (2008). Facilitating adaptive emotional analysis :Distinguishing distanced-analysis of depressive experiences from immersed-analysis and distraction. Personality and Social Psychology Bulletin, 34, 924 -938.doi:10.1177/0146167208315938

[25]. Kross, E., \& Ayduk, Ö. (2009). Boundary conditions and buffering effects: Does depressive symptomology moderate the effectiveness of distanced analysis for facilitating adaptive self-reflection? Journal of Research in Personality, 43, 923-927. doi:10.1016/j.jrp.2009.04.004

[26]. Kross, E., \& Ayduk, Ö. (2011). Making meaning out of negative experiences by self-distancing. Current Directions in Psychological Science, 20(3), 187-191. doi: 10.1177/0963721411408883.

[27]. Kross, E., Gard, D., Deldin, Clifton, P., \& Ayduk, O. (2012). “Asking Why” From a Distance: Its Cognitive and Emotional Consequences for People With Major Depressive Disorder. Journal of Abnormal Psychology, 3, 559 -569. doi: 10.1037/a0028808

[28]. Kross, E., Bruehlman-Senecal, E., Park, J., Burson, A., Dougherty, A., Shablack, H., ...Ayduk, Ö. (2014). Self-talk as a regulatory mechanism: How you do it matters. Journal of Personality and Social Psychology, 106(2), 304.doi: 10.1037/a0035173

[29]. Lievaart, M., Van der Heiden, C., \& Geraerts, E. (2013). Associations between Depressive Symptoms, Rumination, Over general Autobiographical Memory and Interpretation Bias within a Clinically Depressed Sample. Journal Psychology Psychotherapy, S7:004. doi: 10.4172/2161-0487. S7004

[30]. Liberman, N.\& Trope, Y. (2008). The Psychology of Transcending the Here and Now. Science, 322, 1201-1205. doi: 10.1126/science.1161958

[31]. Levin, K.A. (2007). Study design VII. Randomised controlled trials. Evidence-Based Dentistry, 8, 22-23. doi:10.1038/sj.ebd.6400473

[32]. NeshatDoost, H.T., Dalgleish, T., Yule, W., Kalantari, M., Ahmadi, S.J., Dryregrov A., \& Jobson, L. (2013). Enhancing autobiographical memory specificity through cognitive training: an intervention for depression translated from basic science. Clinical Psychological Science, 1:84-92. doi: $10.1177 / 2167702612454613$

[33]. Nolen-Hoeksema, S. (1991) Responses to depression and their effects on the duration of the depressive episode. Journal of Abnormal Psychology, 100, 569-582. doi: 10.1037/0021843X.100.4.569

[34]. Peng, C.Y. J., Harwell, M., Liou, S.M., \& Ehman, L.H. (2006). Advances in missing data methods and implications for educational research. In S. Sawilowsky (Ed.), Real data analysis (pp. 3178). Greenwich, CT: Information Age.

[35]. Raes, F., Williams, J.M.G. \& Hermans, D. (2009): Reducing cognitive vulnerability to depression: A preliminary investigation of Memory Specificity Training (MEST) in inpatients with depressive symptomatology. Journal BehaviorTherapy Experimental Psychiatry, 40, 2438.doi:10.1016/j.jbtep.2008.03.001.

[36]. Raes, F., Hermans, D., Williams, J.M.G., Beyers, W., Brunfaut E., \& Eelen, P. (2006). Reduced autobiographical memory specificity and rumination in predicting the course of depression. Journal Abnormal Psychology, 115, 699-704.

http://dx.doi.org/10.1037/0021-843X.115.4.699

[37]. Sumner, J.A., Griffith, J.W., \& Mineka, S. (2010). Over general autobiographical memory as a predictor of the course of depression: A meta-analysis. Behaviour Research Therapy, 48, 614-625. doi: 10.1016/j.brat.2010.03.013

[38]. Sumner, J.A (2012). The mechanisms underlying overgeneral autobiographical memory: an evaluative review of evidence for the CaR-FA-X model. Clinical Psychology Review. 32, 34-48. doi: 10.1016/j.cpr.2011.10.003

[39]. Suresh, K.P. (2011). An overview of randomization techniques: An unbiased assessment of outcome in clinical research. Human Reproductive Science. 4, 8-11. doi: 10.4103/0974-1208.82352

[40]. Sahin, N., Sahin, N., H., \& Heppner, P. (1993). Psychometric properties of problem solving inventory in a group of Turkish University students. Cognitive Therapy Research, 379-396. doi:10.1007/BF01177661 
Texila International Journal of Psychology

Volume 1, Issue 2, Dec 2016

[41]. Williams, J.M.G., Barnhofer, T., Crane, C., Herman, D., Raes, F., Watkins, E., \& Dalgleish, T. (2007). Autobiographical memory specificity and emotional disorder. Psychological Bulletin, 133,:122-148. doi: 10.1037/0033-2909.133.1.122

[42]. Watkins, E., \& Teasdale, J. D. (2001). Rumination and overgeneral memory in depression: effects of self-focus and analytic thinking. Journal of Abnormal Psychology, 110, 353-357.

[43]. Watkins, E., \& Teasdale, J.D. (2004). Adaptive and maladaptive self-focus in depression. Journal of Affective Disorder, 82(1), 1-8.

[44]. Wegner, D. M., Schneider, D. J., Carter, S. I., \& White, L. (1987). Paradoxical effects of thought suppression. Journal of Personality and Social Psychology, 53, 5-13. http://dx.doi.org/10.1037/0022-

3514.53.1.5

[45]. Williams, J. M. G., \& Broadbent, K. (1986). Autobiographical memory in suicide attempters Journal of Abnormal Psychology, 95, 144-149.doi:org/10/1037/0021-843X.95.2.144

[46]. Wang, Y.P., \& Gorenstein, C. (2013). Psychometric properties of the Beck Depression InventoryII: a comprehensive review. Revista Brasileira de Psiquiatria.35:416-431. doi:10.1590/1516-44462012-1048

[47]. Wegner, D. M. \& Zanakos, S. (1994). Chronic thought suppression. Journal of Personality, 62, 615-640. doi: 10.1111/j.1467-6494.1994.tb00311.x

\section{Funding}

This study was self-funded by the author.

\section{Conflict of Interest}

There are no conflicts of interest for the author 